\title{
Features of Computer Methods in Political Forecasting
}

\author{
Uliana Movchan \\ MA student, Taras Shevchenko National University of Kyiv (Kyiv, Ukraine) \\ E-mail: ekzemplyar6@gmail.com \\ https://orcid.org/0000-0001-6057-3677
}

This paper reveals the essence of computer methods of political forecasting, their classification and characteristics of different groups of methods. Article explore the classification of forecasting methods according to different criteria. We analyze the differences of computer and theoretical (expert) methods. In addition, we explore the advantages of using computer methods in political forecasting.

The purpose of the article is to study the peculiarities of the computer methods application in political forecasting. The principal results: classification of computer methods were created, as well as a detailed study of their features in the process of political forecasting. The major conclusions: forecasting and making its results mathematically clear. These methods provide the opportunity to reuse one model for different situations in political forecasting, unlike theoretical methods. The studying of this problem allows us to discover diverse perspectives of research in political forecasting, namely: the development of new methods of political forecasting, the study of the possibilities of integrating the methods of other sciences into the process of political forecasting.

Keywords: computer methods; political forecasting; political analysis; prognostication

Received: November 18, 2018; accepted: November 30, 2018

Ukrainian Policymaker, Volume 3, 2018: 29-35.

https://doi.org/10.29202/up/3/4

Computer methods - a set of techniques, operations of cognition and practical activities that are used in computer systems, in the form of codes, models, algorithms, programs.

Since the forecasting process is rather complex and involves a lot of constituent elements, different stages are distinguished within different approaches. Foreign researchers distinguish the stages of the overall forecasting process, which are practically oriented [Hyndman \& Athanasopoulos, 2014: 21 - 22]:

1. Determination of the problem;

2. Collecting information about the object;

3. Preliminary (reconnaissance) analysis of the object;

4. Selection and adaptation of forecasting models;

5. Using (testing) and estimation of the predicted model.

(C) Movchan, Uliana, 2018 
This approach, in our opinion, demonstrates the real process of forecasting and it is best correlated with the use of computer methods. Ukrainian researchers use the theoretical approach and distinguish the following stages of forecasting [Gorbatenko \& Butovska, 2005: 23]:

1. Identifying the goals;

2. Obtaining information;

3. Information processing;

4. Assessment and analysis of information;

5. Determination the prospects and probability of the realization of the forecast.

This approach, in our opinion, does not reflect the true nature of the forecasting process. The listed stages can reveal the essence of the political analysis process. However, within the framework of political forecasting, such a wide range of practical methods is used that cannot be used within the specified stages, and, therefore, it is impossible to implement a structured forecast and that affects its reliability.

In terms of «computer», the forecasting process is expressed in the following stages:

1. Receiving, processing and analysis of information about the object;

2. Codification of information, its preparation for forecasting;

3. The process of forecasting;

4. Visualization of the forecast.

Computer techniques can be used at different stages of political forecasting, even if they were not used at the preliminary stages of the forecasting process. Thus, according to the levels of computer methods, political forecasts are divided into the following types:

1. Automated - provide the using of computer methods at all stages of forecasting. Today, most of the political forecasts require human-expert assistance, in particular: to put the necessary data into the system, which will independently carry out an analysis of information for the further forecast. However, special services of different countries already use fully automated systems that can independently collect and allocate necessary information (via the Internet) for further forecasting.

2. Partially managed — provide for the processing and analysis of information through computer systems. Instead, the forecast is carried out directly by a person, an expert, based on the received information. There is also a possible situation where an expert collects, processes and analyzes information on his own, and forecasts are made by using computer methods.

3. Fully managed - provide for using by expert of various theoretical and practical methods and complete rejection of computer methods. At the same time, there is a possible situation when, despite the refusal of computer methods, the visualization of the political forecast is carried out with the help of various modelling systems.

Computer methods solve a significant range of problems that arise in the process of political forecasting. The most important are:

1) Data problems:

a) The presence of a large array of non-homogeneous data necessary for the implementation of the political forecast;

b) Disordered quantitative and qualitative data necessary for the implementation of the forecast.

Due to computer methods, it is possible to clearly arrange the data, to track the patterns and certain trends. 
2) Time problems:

a) Lack of sufficient time for complex forecasting, since the process of analysis of the initial data on the political situation may take more than one month;

b) The need for a long-term forecast (up to 10 years or more).

Computer techniques are able to assist in the rapid analysis of available data, as well as make predictions with high probability for several decades.

3) The problems of alternatives: availability of a large number of possible alternatives to the development of a particular political situation.

Computer methods are able to arrange the possible alternatives to the development of events in «decision trees», that is, in several ways of development simultaneously. The decision tree is a decision support tool used in statistics and data analysis for predictive models.

4) Reliability problems: the presence of a multicomponent political situation, the forecast of which may be subjective.

Due to computer methods, it is possible to analyze all components of a complex political situation with high reliability.

In political forecasting can be used an extremely large number of computer methods. It is worthwhile distinguishing the varieties of the following methods:

1. Analytical. Provide data analysis (due to computer tools), based on which it could be made a political forecast. These include the process of creating Event Data, the implementation of event analysis. In the process of analysis, coding systems are used, namely WEIS, COPDAB, CREON, BCOW, CASCON. Analytical computer methods are used, in particular, to predict the results of a political conflict (using the Levenshtein metric and the "conflict clusters" by F. Schrodt).

2. Modelling. Provide the creation (due to computer tools) of models that can demonstrate the process of leakage and the result of a political phenomenon. This kind of computer techniques is the most popular, since it allows you to make a long-term political forecast and get its graphical, interactive representation. There are different approaches to classify the computer models. Some researchers distinguish the following types: agentoriented (agent) models, evolutionary, sectional, microanalytic simulation, dynamic and related [Benoit, 2001:15]. Also, formalized models (model deprivation, model Polity IV State Fragility Index) can be distinguished; deterministic models (model of state stability MIT, Richardson arms race model, black box model of the political system, differential models of hierarchical control) and agent-oriented models (Epstein model of civil violence). There are also statistical models (Stephenson model of cycles of political mood, models of electoral cycles), but they are applied mainly within the limits of general statistical methods as one of the elements of forecasting [Stevenson, 2001: 620].

3. Statistical. Provide definition (due to computer tools) dynamics of the political process, its trends, cycles and seasonality, as well as the interactions of time series. These include the Technical analysis of the Time Series: smoothing techniques, fitting functions; decomposition of time series: spectral analysis (Fourier analysis); statistical analysis of time series interactions: cross-spectral analysis.

4. Qualitative. It involves the use of the experience, knowledge and intuition of the researcher to carry out the forecast, as well as the use of computer tools for visualizing this forecast. These types of methods include: the Delphi method and the method of brainstorming. However, their application in computer systems is inappropriate, since 
they do not provide mathematical precision of the results. Instead, as a computer method in science, the construction of scenarios is actively used. It is worth highlighting the deductive-inductive method of constructing political scenarios, as well as constructing scenarios based on situational analysis.

Most of the theoretical or expert methods of political forecasting today are used by a number of computer methods to systematize data and visualize them. Thus, the group of computer methods of political forecasting is increasingly expanding at the expense of the evolution of expert methods.

Among the analytical computer methods in political forecasting, event analysis is the most used. Event analysis or analysis of event data is a method of studying the dynamics of political situations, which is based on monitoring the intensity of events in order to identify possible ways of their evolution, as well as identify the components of the political process, which are the most important for research. However, gradually, in the process of political forecasting, begin to use more sophisticated analytical methods based on a variety of encoding systems and programs. Today, the following methods are used: Survival Analysis (Paul Dil, Janet BoxSteffensmeier) - a method of investigating the probability of occurrence of the event; Rare events analysis (Gary King) - a method of analyzing individual political events, and not their aggregates; Neural Networks (Langche Zeng) - a method for finding purposes and means of treatment in a wide range of environments (in particular, political), based on simulating the work of human intelligence; Social / Geographic Network analysis (Michael Ward, Kristian Gleditsch) - a method of researching social relationships within a particular social structure created by individuals or organizations; the Bayesian method (John Freeman, Patrick Brundt) is a method of making decisions about unobservable characteristics, based on knowledge of the a priori distribution of the probabilities of these characteristics and on the conditional distribution of experimental results for given values of unobservable characteristics; Cluster Analysis (Robert Trapple) is a multi-dimensional research method, which includes the collection of data that contains information about sample objects, their arrangement in relatively homogeneous, group-like, and group-based analysis.

The following analytical models are also used: the Heckman model, which includes two variables, that allows to determine the intensity of political participation of individuals, as well as to analyze the factors of their non-participation; Hidden Markov Model is a random process generated by two interrelated stochastic mechanisms, these Markov chains having a finite number of states and a set of random functions, each of which is associated with a corresponding state; Wolfram Reversible Models - a platform for mathematical and computer modelling objects and systems based on the language Modelica; Poisson's Regression Model - a method for describing rare events: includes a series of random events occurring over a fixed period of time or in a fixed area of space. Computer analytical methods are popular in prognostic studies, most of which are perennial or permanent.

Among the computer modeling methods, it is worthwhile to distinguish the following classifications.

By the type of language in which the models are formulated - meaningful and formal. By functionality, content models are divided into descriptive, explanatory and prognostic ones. Among the predictive, the most common are a variety of mathematical models. In general, they can be divided into three interrelated groups:

1. Deterministic models presented in the form of equations and inequalities that describe the behavior of the object being studied; 
2. Optimization models that contain a certain expression that needs to be maximized or minimized under certain constraints;

3. Probabilistic models, which are also expressed in the form of equations and inequalities, but have a probable meaning, that is, finding a solution is based on maximizing the average utility value.

At logical levels, models are divided into macro- and micro-models. Depending on the method of describing the object of the model, quantitative and qualitative [Yakovlev, 1998: $15]$.

As concerns to reality, there are distinguished present, possible and desirable state of the system. The first ones are used in the study of the properties of a real object. Models of the second and third type are formed if necessary taking into account possible changes of the given object under the influence of various circumstances. The model of the problem situation is used, when there is a contradiction between the data and the state of the system. Ways and means to overcome this contradiction are contained in decision models.

In general, the essence of the modelling consists in replacing the real object of political reality with the object $\mathrm{B}$ created artificially, and the one that repeats the essential aspects of the object A, that is, its model. A model is an image of an object or structure, an explanation or description of a system, process or series of related events. For the simulation of any structure, object or process, a system of equations is formed. Communication systems within the models are presented by compiling a flow diagram of information flow, for example, by mathematical or logical-semantic modelling.

In the construction of mathematical models, mainly linear programming, dynamic programming, graph theory are used. However, when solving problems relating to the study of a political object, researchers often stop at forming a model without carrying out a detailed study of it. Different methods of modelling are used for solving research problems. The most developed is the systematic approach, which allows to consider the object of research as a system. Within the system approach, we use content models, namely: models of crises, revolutions, catastrophes, chaos. The Theory of Rational Choice, Downness Election model are also actively used. Political modelling combines mathematical techniques (Linear Modeling, Geometric method of Medulation, Graph Theory, Mynamic modeling); physical and chemical techniques (Models of Chaos, Evolution); psychology Models of Conflicts); economic methods (econometric methods, Theory of Decision Making, Analysis of Economic Behavior). In recent years, the Hierarchy Analysis method, developed by Thomas Saaty, is used. Scientists use different systems for evaluation of the models. For example, there are GDELT interaction graphs.

Computer modelling methods are characterized by a significant number of benefits when applied in the process of political forecasting. Much of the events in political life is expected, so its appearance can be foreseen. The models help to express such informal predictions. Secondly, the formal model helps to overcome the free formulations of the assumptions of the informal model and provide a more accurate prediction that can be verified. Thirdly, the advantage of formal models is their ability to systematically operate concepts and elements of a higher level of complexity.

Most complex systems models are not particularly aimed to foresee the future state of the world. On the other hand, that is precisely the main goal of most global models: to provide a forecast of the future state of the world, or parts of it, using (perhaps many) mathematical equations and assumptions [Castro \& Jacovkis, 2015]. 
Computer statistic methods are scientific methods for describing and studying mass phenomena using computer programs and systems that allow quantitative (numerical) expressions. Today, there are comprehensive groups of computer statistical methods known as packets. One of the most popular statistical packages is SPSS. It combines several dozen different statistical and analytical methods (for example, the survival analysis described above). Most statistical packages are based on Time Series analysis. Also there are popular packages such as: STATISTICA, SAS, STATGRAPHICS PLUS, Systat. The complex statistical packages provide the ability to author for development of computer applications that allow the prediction certain types of political phenomena, such as: Stata, MESOSAUR, STADIA, EViews. Despite the variety of statistical methods, in the post-Soviet space, the MicrosoftExcel software package is used predominantly.

As it was mentioned, computer high-quality methods are characterized by the lowest level of use of technological tools. However, the best examples of combining a qualitative method with modern technological tools is the Scenario method and the Theory of Moves. The method involves the creation of scenarios that provide a high probability of working out effective solutions in situations where this is possible and higher probability of reducing expected losses in situations where losing is impossible. The Scenario method includes search and regulatory scenarios [Henrichs, 2003].

Over the past years, we see that computer methods are increasingly becoming used not only for the «private» forecasting of political events and processes, but also for the public. There has been an effort to create a forecasting platform for decision-making within the defense and intelligence communities, again based on open sources. For example, this is W-ICEWS, the Worldwide Integrated Crisis Early Warning System, often abbreviated ICEWS. It has a lot of components, but the most pertinent is the suite of models, developed by social scientists, that forecast major instability events around the world with high accuracy. The basic idea is to use data that are detailed but aggregated to the month. These data are both structural and behavioral. The behavior data are event data produced using a tested ontology of categories and an automated procedure for constructing word graphs of stories order to glean context [Ward, 2016].

Despite the fact that computer methods are already being used in the process of political forecasting in many countries of the world, they have significant further prospects for development and implementation. Each year, computer methods undergo modifications, and the modern technologies allows the development entirely new methods. It will be possible soon to allocate a separate group of computer methods: the online methods of forecasting. Certain bases for the formation of such methods were laid in 2011 when Research Associates, Inc. started testing the ACES prediction system. This system gave citizens the opportunity to participate in the crowdsourcing intelligence of the future. The fundamental novelty of the system is that forecasts are processed on the basis of special computational models that can improve human hypotheses, and prevent them from bias and stress influence. The project is funded by the Office of the Director of National Intelligence (IARPA) [Rawnsley, 2011].

The computer methods used in political forecasting completely differ from the corresponding theoretical (expert) methods. According to Philip Schrodt, computer methods are most effective in predicting the development of a global political situation or problem, but they can not be used to predict individual political events. To predict such events, it is necessary to apply theoretical (expert) methods.

Comparison of computer and theoretical (expert) methods [Schrodt \& Yonamine, 2013: $18]$. 


\begin{tabular}{|l|l|}
\hline Computer methods & Theoretical (expert) methods \\
\hline Objective & Subjective \\
\hline Rigid / stable & Flexible \\
\hline $\begin{array}{l}\text { Inability to study each individual event } \\
\text { (study only a set of events) }\end{array}$ & Ability to interpret each individual event \\
\hline $\begin{array}{l}\text { Minimal and one-time financial costs of } \\
\text { applying methods }\end{array}$ & $\begin{array}{l}\text { Constant financial expenses for application of } \\
\text { methods }\end{array}$ \\
\hline $\begin{array}{l}\text { The impossibility of predicting new } \\
\text { phenomena }\end{array}$ & Ability to predict new phenomena \\
\hline
\end{tabular}

Thus, computer methods have an extremely wide range of opportunities for political forecasting. Already in the coming years, computer methods will promote the fact that the process of political forecasting will be carried out not only within special structures or research institutions, but also by any ordinary person.

\section{References}

Benoit, Kenneth. Simulation Methodologies for Political Scientists. Computational Modeling. The Political Methodologist. Vol. 10. №1, 2001: $12-16$.

Castro, Rodrigo, and Pablo Jacovkis. Computer-Based Global Models: From Early Experiences to Complex Systems. Journal of Artificial Societies and Social Simulation. Vol. 18 (1), $1,2015$.

Gorbatenko, Volodymyr and Iryna Butovska. Political Forecasting: Tutorial. MAUP, 2005.

Henrichs, Thomas. Scenarios: Environmental Scenario Analysis, Overview and Approaches. 2003. URL: http://www.gecafs.org/gecafs_meetings/2003_08_18/

Hyndman, Rob J, and George Athanasopoulos. Forecasting: principles and practice. Otexts, 2014.

Keneshloo, Yaser, Jose Cadena, Gizem Korkmaz, and Naren Ramakrishnan. Detecting and Forecasting Domestic Political Crises: A Graph-based Approach. WebSci '14 Proceedings of the 2014 ACM conference on Web science, 2014. URL: http://people. cs.vt.edu/naren/papers/websci-gdelt-2014.pdf

Rawnsley, Adam. Spy Agency's Next Top Analyst. 2011. URL: https://www.wired.com/2011/07/ spy-agencys-next-top-analyst-you

Schrodt, Philip and Jay Yonamine. A Guide to Event Data: Past, Present, and Future. All Azimuth. Journal of Foreign Policy and Peace. Vol. 2. №2, 2013: 5- 22.

Stevenson, Randolph. The economy and policy mood: a fundamental dynamic of democratic politics? American Journal of Political Science. №45 (3), 2001: 620 - 633.

Ward, Michael D. Can We Predict Politics? Toward What End? Journal of Global Security Studies. Vol. 1. №1, 2016: $80-91$.

Yakovlev, Igor. Information-Analytical Technologies and Political Consulting. Polis. №2, 1998: 122. 